\title{
L'écologie au service du projet de paysage : réinventer une pensée paysagère?
}

\author{
Ecology at the service of the landscape project: reinventing landscape \\ thinking?
}

\author{
Yves Petit-Berghem ${ }^{1}$ \\ ${ }^{1}$ LAREP, École Nationale Supérieure de Paysage (y.petitberghem@ecole-paysage.fr)
}

\begin{abstract}
RÉSUMÉ. Science d'observation par excellence, l'écologie est progressivement devenue une discipline attachée aux normes, aux microscopes, aux modèles virtuels abstraits éloignés de la réalité du terrain. Lorsqu'il s'agit d'aborder l'étude des paysages, l'écologie peine à formaliser son champ de connaissances de manière à acquérir une véritable dimension opérationnelle. Elle a pourtant toute sa place dans les écoles formant les paysagistes dont les pratiques consistent à imaginer un nouvel environnement spatial, en intégrant les données de la nature combinées à celles produites par les sociétés. Elle participe également au renouvellement des modes de représentation paysagère en proposant de nouvelles approches adaptées à la démarche de projet. C'est dans la discussion et par le raisonnement que les représentations participent à la construction d'une pensée paysagère complexe et d'un savoir qui s'enrichit par l'action.

ABSTRACT. Initially based on observation, ecology has gradually become a discipline attached to standards, microscopes and abstract virtual models that are far from the reality. When it comes to the study of landscapes, ecology struggles to formalize its field of knowledge in order to acquire a real operational dimension. Yet it has its full place in schools training landscape designers whose practices consist in imagining a new spatial environment, by integrating data from nature combined with those produced by societies. Ecology also participates in the renewal of landscape representation methods by proposing new approaches adapted to the landscape project. It is through discussion and reasoning that representations participate in the construction of a complex landscape thinking and knowledge that is enriched by action.

MOTS-CLÉS. écologie, paysagiste, représentations paysagères, projet de paysage, pratiques pédagogiques.

KEYWORDS. ecology, landscape design, landscape representations, landscape project, pedagogical practices.
\end{abstract}

\section{Introduction}

Dans les écoles formant les paysagistes, l'écologie constitue un substrat à partir duquel naissent des approches nourrissant des projets (Chauvel et Rumelhart, 1988 ; Petit-Berghem, 2016) dont la finalité est de proposer des solutions originales qui ont valeur d'exemples. Selon cette logique, l'établissement d'un projet est toujours dépendant de faits de nature régis par des lois à portée universelle décrites par les sciences. Quelques rares recherches prenant des réalisations paysagères comme cas d'étude (Mollie, 2009 ; Arnould, 2012) ont d'ailleurs fait état de quelques modalités de création des «nouveaux écosystèmes » (Lévêque, 2017) générés par la pratique des paysagistes. Parfois proches des systèmes historiques, ou bien complètement nouveaux (Hobbs et al., 2009), ces écosystèmes naissent de conceptions par lesquelles les matérialités vivantes sont prises en compte au prisme du fonctionnement de leurs milieux. Cette recherche de faits facilite le travail de reconnaissance des lieux et des dynamiques qui les animent. A ce titre, l'écologie, par ce qu'elle produit, imprime sa marque dans le paysage ; elle façonne à la fois son apparence globale (sa physionomie) et ses nuances dans l'expression de ses formes ou de ses motifs.

Pour montrer ces paysages et les représenter graphiquement, l'écologie s'appuie sur des outils de représentation complémentaires de ceux utilisés en géographie. Aussi, les cartes, blocs-diagramme, photographies au sol ou aériennes, images satellites sont couramment utilisés pour représenter les 
espèces, les structures paysagères, les relations entre les sols et les couverts végétaux. C'est à la fois le paysage dans sa matérialité physique et biologique qui retient l'attention que les milieux à partir desquels des systèmes anthropisés ont été mis en place par les sociétés. La co-évolution hommenature explique en effet que les paysages résultent d'un ensemble interactif société-milieux naturels qui a justifié l'émergence des notions d'écosystème puis de géosystème.

Les projets de paysage se nourrissent de ces représentations qui expriment aussi une façon de concevoir un espace que le paysagiste personnalise en exprimant sa sensibilité et en opérant des choix qu'il assume pour défendre un projet. L'écologie lui offre également la possibilité d'utiliser des représentations savantes construites notamment dans le cadre des systèmes d'informations géographiques (SIG). Les données traitées sur ordinateur produisent un paysage propre à la mesure et à la généralisation, et donc aux représentations géométriques d'un espace fini qui anéantit toute incertitude ou imaginaire. Une tension peut alors naître entre le paysage inventé, individualisé, fruit d'une pratique ou d'un art, et le paysage numérique, fruit d'une pensée rationnelle et d'une démarche scientifique. Dans les écoles formant les paysagistes, il importe aux formateurs d'apaiser cette tension en montrant la complémentarité de ces représentations et en questionnant l'évolution des modes d'apprentissage que cette situation induit.

\section{Ecologie et projet de paysage}

\subsection{Ecologie et sciences du paysage}

L'écologie, en tant que discipline scientifique, se définit dès le XIX ${ }^{\mathrm{e}}$ siècle comme une science centrée sur les êtres vivants ; l'objectif est d'étudier ces êtres vivants et le milieu (organique, inorganique) dans lequel ils vivent. Cette écologie implique des apports dans les sciences connexes, que celles-ci relèvent des êtres vivants végétaux ou animaux que des milieux envisagés dans leur complexité physico-chimique et organique. Dès le départ, l'écologie se donne pour mission d'inventorier, de qualifier des milieux, et aussi de les spatialiser (Lévêque, 2013). Cette écologie s'intéresse peu aux paysages sauf ceux apparaissant sur de larges étendues, aisément reconnaissables par leur physionomie, et dont la distribution est étroitement liée à celle des climats. Ce sont des biomes, puis par un glissement d'échelle, des écosystèmes lorsque le terme est créé par A.-G. Tansley en 1935. À la même époque, l'écologie du paysage apparaît en Allemagne et envisage le paysage comme un complexe d'écosystèmes fragmentés par les activités humaines. Cette écologie se diffuse relativement peu en France jusqu'aux années 1970 car la biodiversité n'est pas encore un sujet de préoccupation et encore moins un objet scientifique. Parallèlement, les géographes, en particulier ceux qui gravitent autour de G. Bertrand, renouvellent la pensée écologique du paysage en considérant qu'il faut l'aborder et l'étudier de façon systémique. Le concept du géosystème, introduit par la science géographique soviétique, s'inspire des écosystèmes des biologistes ; il a pour objectif d'analyser les combinaisons dynamiques de facteurs biotiques, abiotiques et anthropiques associés. Théorisé par G. Bertrand à la fin des années 1960 (Beroutchachvili et Rougerie, 1991), il permet de relier toutes les variables de l'environnement et d'envisager un fonctionnement à différentes échelles accordant une place privilégié à l'homme et aux sociétés. En saisissant la complexité des milieux, les fonctionnements et les dynamiques, le géosystème « révèle » le paysage en montrant que tout s'intègre, et que cette complexité est le fruit d'une combinaison de variables elles-mêmes variables, c'est-à-dire évolutives dans le temps.

Ce sont les paysages végétaux qui retiennent le plus l'attention, leurs formes, leurs couleurs, en particulier lorsque ceux-ci s'imbriquent dans des séquences paysagères zonées ou étagées selon des gradients climatiques. Aussi, sur le terrain, les observations conduisent les phytogéographes à mettre en relation ces formes avec des facteurs écologiques liés aux lois de la nature ; cette recherche de 
rationalité n'exclut cependant pas une dimension plus sensible où la forme est aussi celle de l'inventivité du vivant, sa capacité à s'adapter aux conditions ambiantes et à provoquer des émotions comme l'avait d'ailleurs déjà montré Alexander Von Humboldt, dans Cosmos ou dans son Essai sur la géographie des plantes ${ }^{1}$. La lecture de ces formes renseigne sur l'état du milieu ou alors sur des rythmes du vivant, imposés par la nature ou par l'homme. En forêt, ces rythmes permettent par exemple de voir la régénération d'une plante puis sa croissance et son intégration progressive dans le programme des coupes lié à l'exploitation de cette forêt. Le paysage forestier est aussi marqué par l'enchevêtrement de temps, un temps long lié à la durée de vie des arbres mais un temps qui peut aussi brusquement s'accélérer lorsqu'un événement programmé (par exemple une coupe) ou fortuit (par exemple une tempête) arrive.

Ces manières d'appréhender les paysages se sont complexifiées au fil du temps, dans la mesure où les systèmes ont intégré des enjeux liés aux services environnementaux et aux difficultés d'une gestion multifonctionnelle renvoyant à des acteurs variés pouvant entrer en conflit.

En donnant au système une dimension plus anthropocentrique, 1'approche des milieux bifurque progressivement vers une approche plus territoriale et sociale ; cette évolution est en phase aussi avec une volonté d'aller vers le paysage territorialisé, qui est pour beaucoup de géographes une coproduction entre les données de nature et l'œuvre des hommes. En cela, le géographe s'inscrit dans la volonté de saisir ces paysages dans leur trajectoire évolutive, de comprendre pourquoi certains évènements sont importants à la fois dans la genèse de nouveaux paysages mais aussi dans leur perpétuelle transformation. C'est une différence avec les écologues qui parlent aussi de trajectoires mais en recherchant des référents dans l'histoire par rapport à un certain état d'un écosystème qui pourrait servir d'exemple, comme pour envisager la restauration d'un milieu.

Le projet de paysage empreinte donc aux sciences - écologiques et géographiques ici - des éléments théoriques, des concepts et notions issus d'un savoir savant qui s'est constitué au fil du temps. Cette démarche du projet se nourrit également d'un savoir vernaculaire construit par l'expérience et l'expression de multiples sensibilités.

\subsection{Sciences et sensibilités}

Une approche scientifique n'est pas incompatible avec une approche sensible laissant libre court aux expériences, aux intuitions et à la création. En effet, si la science repose sur des faits et l'explication aux phénomènes que nous percevons, elle tend souvent - par la théorie ou la doctrine à établir des modèles de la réalité. La science permet de comprendre mais elle ne place pas l'individu au centre de la réflexion. Or, le paysage n'est pas qu'une enveloppe matérielle dépersonnalisée dont on cherche à comprendre le fonctionnement, il est aussi sujet à des constructions mentales et à des observations qui permettent à tout à chacun de se faire sa propre réalité indépendamment de tout modèle présupposé ou établi. Le paysage invite donc à l'immersion, car l'être humain ressent et fait l'expérience de son corps et, par son intermédiaire, de la réalité sensible. Par sa physionomie, ses couleurs, ses sons, un paysage de nature est susceptible d'éveiller en nous les organes sensoriels permettant de le percevoir. Ce paysage nous parle mais pour l'entendre, nous devons l'écouter, c'est-à-dire observer le développement des phénomènes, sans en chercher forcément la signification. Puisque la science donne une explication aux couleurs, senteurs, textures, et sons de la nature, elle doit aussi permettre aux hommes de s'en abstraire afin de faciliter cette immersion paysagère. C'est en procédant de la sorte que l'être humain ressent et la nature lui 
apporte des signaux provenant d'un corps et d'un monde extérieur inconnus. Il s'invente alors un monde sensoriel avec des sons, des couleurs, des odeurs, des goûts, qui participent à la fabrication d'ambiances sensorielles (figure 1).

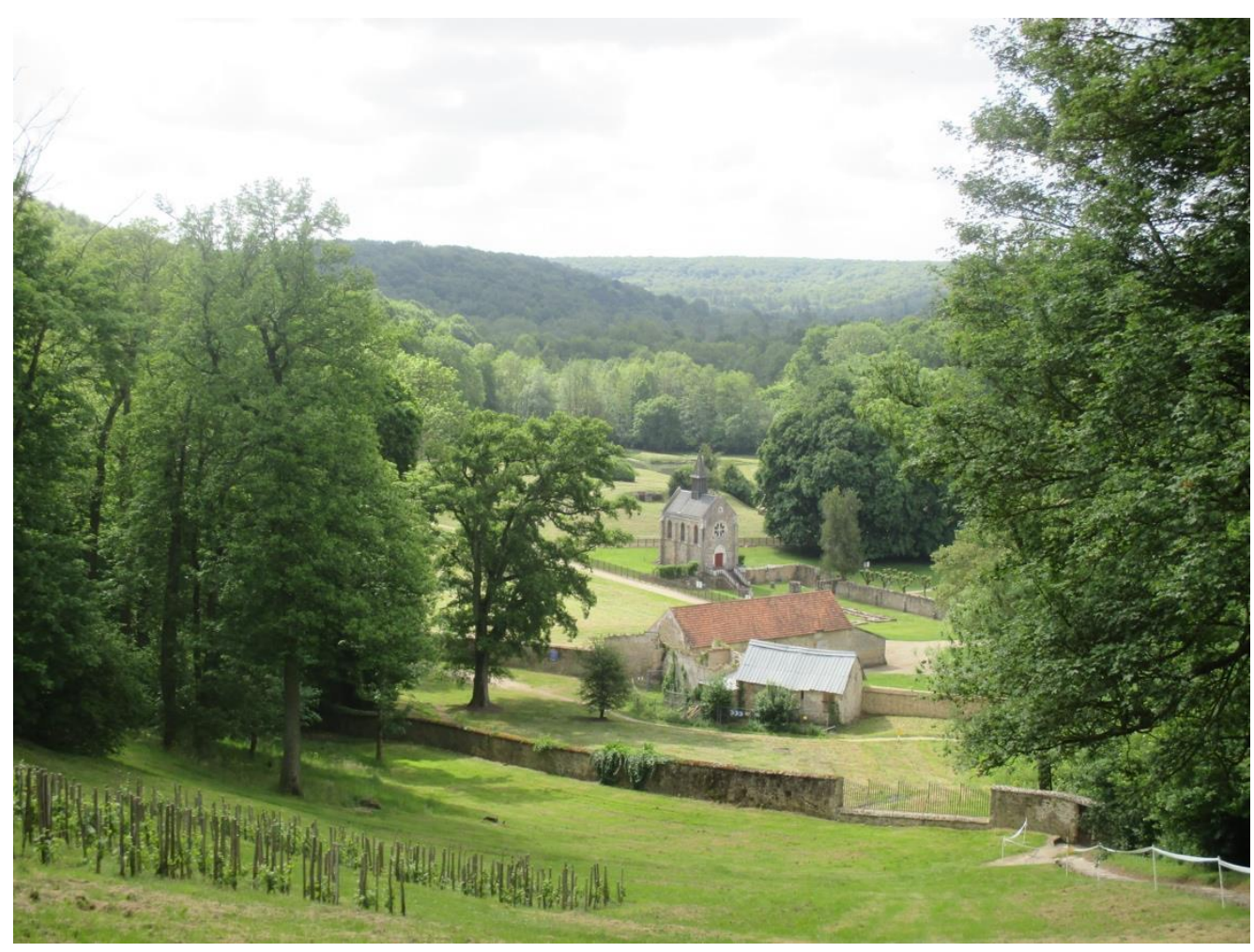

Figure 1. La forêt et l'immersion paysagère Site de Port-Royal (Département des Yvelines, septembre 2016)

En forêt, chacun fait corps avec le paysage en se fondant dans l'élément et en activant ses sens afin d'éveiller en lui des ressources inédites et des émotions. Le langage du corps rend la perception polysensorielle : la forêt distille des odeurs, produit des sons qui se déclinent au fil des saisons, héberge une nature réifiée en permanence qu'il est possible de toucher pour s'en imprégner et en apprécier sa saveur. Ce sont les sens qui font du paysage forestier une entité vivante que l'on peut mettre en scène, une sorte de spectacle à ciel ouvert avec une musicalité douce ou qui s'emballe, des odeurs qui attirent ou qui révulsent, des arbres qui effraient ou que l'on a envie de caresser (Mottet, 2017). La lecture du paysage cristallise des moments où se confrontent l'information visuelle à la présence physique où le tactile dévoile des fractions d'apparences. C'est en s'imprégnant des lieux que le paysage sensible se construit. Cette imprégnation confère au paysage son existence et la valeur que chacun lui accorde. Cette valeur dépend des affects et des sensations qui se dégagent à la lecture de ce tableau. Cette valeur est toute personnelle et diffère de celle attribuée par les sciences qui répond à des normes, des indicateurs que l'expert applique pour gérer un écosystème qu'il faut évaluer et maîtriser. L'appropriation du lieu permet à chacun d'en faire son propre terrain d'expériences, une mise en pratique salutaire, véritable écologie du lieu où les singularités locales du site sont en rupture avec les rationalités existantes basées sur des modèles imposés. Un espace de tensions s'ouvre alors entre la culture individuelle requalifiant par la pratique le lieu et la culture universelle définissant des protocoles d'action, des outils de référencement et des schèmes d'organisation prédéfinis. Cette tension entre modèles de référence et terrains d'expériences n'est pas un problème mais peut susciter une incompréhension quand un espace est trop codifié et réglé par des usages imposés par la réglementation ou une nécessité de gestion.

L'approche scientifique du paysage renvoyant à des formes de matérialité construites et pensées avec le vivant ne doit donc pas faire oublier toute la dimension sensible et idéelle du paysage 
porteuse d'un certain imaginaire, d'un ailleurs dépassant les frontières. Lorsque l'immatérialité l'emporte, le paysage est alors construit par la pensée instinctive de son auteur qui souvent s'échappe des règles et des repères sociaux.

L'artialisation du paysage (Roger, 1997) en est la parfaite illustration, elle s'accompagne de représentations artistiques et d'expériences sensibles où la nature devient un territoire de méditation, de réflexion et de liberté. En forêt, ces sensibilités esthétiques émergent dans la seconde moitié du $\mathrm{XIX}^{\mathrm{e}}$ siècle et préfigurent les mouvements de contestation d'un modèle productiviste et industriel de la foresterie dont l'une des traductions paysagères correspond aux plantations de résineux. Dans ce contexte économique et social, le reboisement apparaît comme une panacée mais il est aussi porteur d'images négatives ou vides de sens pour les artistes attachés à l'esprit des lieux (Arnould, 2014 ; Kalaora, 1981). A Fontainebleau, les artistes revendiquent la conservation des vieilles futaies pour leur caractère esthétique et patrimonial, un refuge symbolisant le libre jeu des forces naturelles, en contraste avec les aires habitées et les surfaces intensément exploitées par l'agriculture.

Dans les faits, ces dimensions matérielles et immatérielles se combinent et se retrouvent aujourd'hui dans la définition même du paysage qui renvoie selon la Convention européenne du paysage à une « partie de territoire tel que perçu par les populations et dont le caractère résulte de la combinaison de facteurs naturels et/ou humains et de leurs interrelations » (art.1a). Le paysage est ainsi producteur d'images qui sont à l'interface entre une réalité visible et le sujet qui le regarde (Luginbühl, 2012). Et puisque les chercheurs en ont fait une représentation territorialisée de l'environnement, le paysage objet est aussi un paysage sujet : il se construit par le relationnel avec des êtres sensibles et non uniquement par des observateurs simplement préoccupés par ce qu'ils voient et souvent tentés par la recherche de liens de causalité.

En articulant la réflexion sur ce continuum de la relation sujet/objet sans chercher à ne privilégier ni l'un ni l'autre, le paysage devient un medium permettant de se rapprocher des hommes et d'établir les bases d'un projet collectif.

Cette orientation plutôt ouverte du paysage en phase aussi avec celle affichée par le Conseil de l'Europe laisse libre court aux modes d'expression et aux outils qui vont être mobilisés dans la pratique pour représenter les paysages, leur écologie et leur dynamique. Quels sont plus précisément ces outils et que nous montrent-ils ?

\section{Ecologie et représentation paysagère}

\subsection{Diversité des représentations}

Un projet de paysage naît de la rencontre avec un concepteur et des acteurs qui interagissent dans la production d'une œuvre collective. Dans cette aventure, un certain nombre de documents sont utilisés et fabriqués. Parmi ces documents, les représentations graphiques ont une place importante car elles accompagnent tout le processus de projet et participent à sa communication. Evoquer ces représentations, c'est aussi se plonger dans un rapport entre un individu et son espace qu'il personnalise. Cet espace est lui-même porteur d'intentions et est à l'origine de choix très personnels qui révèlent la spécificité d'une pensée et souvent sa non reproductibilité.

En effet, dans sa formation, l'apprenti concepteur navigue dans l'espace sensible des affects et des percepts : le paysage n'est pas tant son objet d'étude qu'un regard porté sur le monde qui l'entoure (Corajoud, 2010). La description et la représentation d'un paysage traduisent ainsi un rapport intime entre un être humain et son environnement. Ce regard singulier doit toutefois s'adapter à des commandes publiques qui se renouvellent et à une écologisation des pratiques 
imposée par de nouveaux enjeux écologiques et sociétaux. Les représentations ne doivent donc pas être déconnectées d'une demande territoriale et sociale mais au contraire s'attacher à répondre à cette demande en proposant des solutions innovantes et adaptées.

Traditionnellement, les concepteurs utilisent un certain nombre d'outils pour mener à bien leurs projets. Ces outils leur permettent de tester différents projets d'aménagement ou de gestion d'un espace, voire de communiquer sur un projet plus avancé. Nous avons une grande variété d'outils, allant de la carte institutionnelle ou sensible, aux dessins et blocs-diagramme réalisés à la main, la photographie paysagère au sol ou aérienne, l'œuvre d'art, le schéma analytique ou systémique...Tous ces outils sont dans un entre-deux : entre l'observateur du paysage qui donne corps à sa représentation et les acteurs du paysage qui reçoivent l'information et parfois contribuent aussi à la produire.

Dans les écoles de projet où l'atelier constitue le socle de la pédagogie, le dessin reste aujourd'hui un outil important. Il constitue même le fondement d'une profession qui s'attache à observer des paysages et à les retranscrire sur le vif. Les motifs constituant la charpente paysagère sont mis en scène à l'image des peintres impressionnistes qui s'en allaient peindre sur le motif (Clément et al., 2016). Le dessin est le propre d'une démarche sensible, intuitive, où le regard actif permet souvent de capter la réalité en mouvement. En écologie, ces dessins permettent de retracer un itinéraire, de préciser l'anatomie d'une plante, de représenter la variété des milieux, des faits de nature (arbre, oiseau, rivière), de mettre en scène des objets matériels (sols, animaux) ou immatériels (des acteurs, une pensée). Le dessin figuratif reste aujourd'hui l'outil favori du paysagiste ; il est associé au travail en atelier où les esquisses de projet s'accompagnent de planches de dessin dessinant les paysages de demain. Savoir dessiner sur le motif, en plan, ou en perspective est un atout pour l'apprenti paysagiste qui pourra mettre cette qualité en valeur lorsqu'il sera en situation professionnelle.

Mais si le dessin est utile pour représenter des détails qu'un autre document ne montrerait pas, il est toutefois empreint de subjectivité à moins qu'une légende suffisamment explicite l'accompagne. Cette subjectivité se retrouve encore plus sur le dessin peint à l'aquarelle (figure 2).

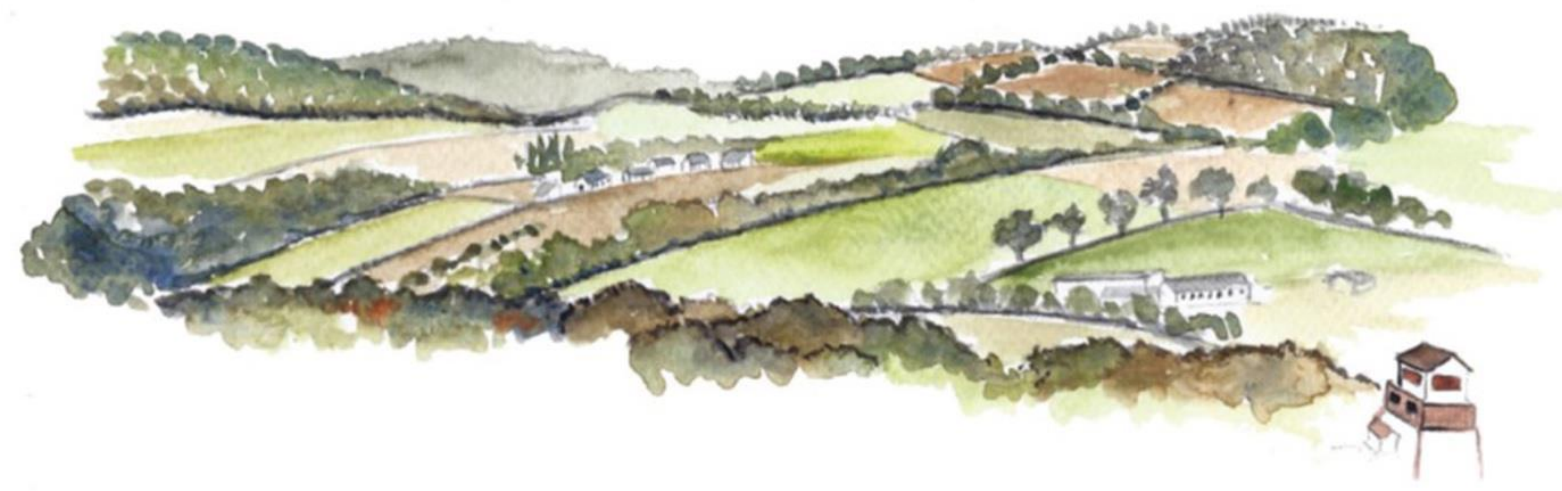

Figure 2. L'aquarelle et la dimension sensible du paysage

Atelier « Diagnostic territorial et analyse paysagère : le canton de Thury-Harcourt (département du Calvados) ". Séquence pédagogique ENSP Versailles $2^{e}$ année (octobre 2015).

Ces dessins sont souvent à l'origine d'un certain « imaginaire paysager » et permettent en écologie de faire ressortir des variables plastiques (couleur, texture) ou des variables d'intensité (lumière, mouvement). Mais, en dépit de sa capacité à fabriquer une scène, à montrer des détails et des contrastes, le dessin n'est pas une représentation offerte à tous, selon des règles convenues ; il 
est ainsi souvent plus ou moins décalé par rapport au réel, en raison des biais individuels et sociaux, et des distances. On sait notamment que les espaces familiers se représentent plus amples et avec plus de détails que les autres (Brunet et al., 1992). Il existe en fait une infinité d'interprétations et de significations possibles pour un même récepteur. La difficulté est que le dessin ne codifie pas l'information qu'il est censé transmettre dans un projet.

D'autres formes de représentation paysagère sont plus abouties et permettent de spatialiser des informations en rendant compte de la diversité des éléments constituant à méso ou grande échelle (1:25 000, 1:10 000) la structure paysagère d'un territoire infrarégional. C'est par exemple le cas des blocs-diagramme dont la construction résulte d'un croisement de techniques entre la carte, les coupes et le dessin perspectif (Michelin, 2000). La géographie vidalienne a utilisé ce type de représentation polygonale montrant en particulier la relation entre le relief et la couverture végétale. Le bloc diagramme a vocation à donner une interprétation synthétique du paysage. Contrairement au dessin, il n'est pas une représentation d'un paysage réel pris sur le vif ; il est levé sur le terrain puis retravailler en salle car il doit donner la ou les structure(s) paysagère(s) du lieu considéré en évacuant le détail et en éliminant l'anecdotique. Cet outil peut exprimer également des dynamiques paysagères, soit en mettant en relief les signes visibles d'évolution, soit en présentant une évolution diachronique en plusieurs étapes. Combiné à la photographie aérienne verticale, le bloc-diagramme permet d'analyser des changements, de voir les invariants, mais son emploi est conditionné à l'emploi d'une échelle particulière qui limite son usage et sa lisibilité. Aussi, son utilisation sera souvent très ciblée, par exemple mettre en évidence la dynamique des couverts végétaux sur un versant et/ou exprimer une intention de projet comme l'illustre la figure 3 définissant un projet d'ouverture de la végétation associé à la mise en valeur de pierriers et à la création de cheminements en haut de versant pour mettre en perspective le paysage de la vallée. Le bloc-diagramme peut être judicieusement combiné à la photographie paysagère dont les avantages ont été maintes fois rappelés dans la constitution des observatoires photographiques de paysage, initiés par le ministère de l'environnement à partir des années 1990. Si ces observatoires permettent de travailler sur la définition des paysages et leurs évolutions, les difficultés d'appréciation peuvent surgir avec le problème des glissements d'échelles, des paysages masqués, d'étendues non délimitées précisément. Les facteurs techniques (angle de prise de vue, focale) donnent des visions très différentes selon les choix effectués par l'opérateur. Ces limites s'estompent lorsque nous avons des documents graphiques dont certains tendent vers une généralisation.

Le recours aux représentations cartographiques institutionnelles est de ce fait courant lorsqu'il s'agit d'appréhender un site, de se repérer. Les règles élémentaires du langage cartographique permettent rapidement de voir les limites des représentations conventionnelles car la carte n'est pas le paysage mais une image des nombreuses interprétations que l'on peut attribuer à un espace. Aussi, les paysagistes ont tendance à se détourner du langage trop formaté des cartes institutionnelles pour inventer d'autres représentations fondées sur un alphabet de l'espace qui leur est propre : des figurés inventés non répertoriés dans les nomenclatures, des codes de couleurs non conventionnels, des limites effacées ou exagérément grossies...autant de signes associés à un langage propre d'un sujet sensible à un espace géographique. La carte sensible est celle des entités et des ambiances paysagères reconstituées pour rendre compte plus fidèlement des rapports du spectateur au paysage non médiatisés par la connaissance savante. Cette carte sensible ne ressemble pas à la carte numérique utilisée pour importer des données, caler une image dans un référentiel terrestre, ou encore interroger des référentiels existants. 


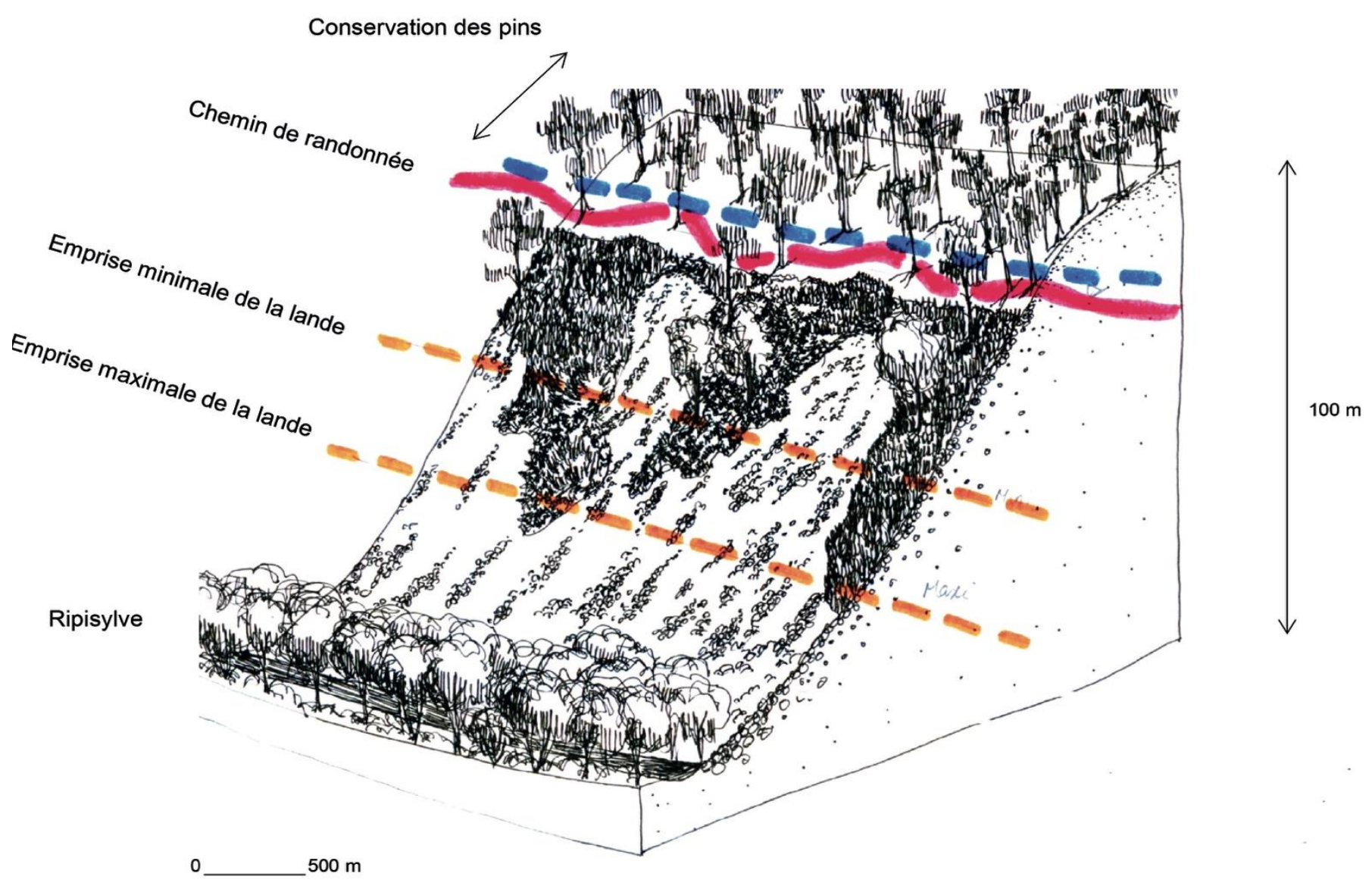

Figure 3. Bloc diagramme et esquisse de projet

Atelier «Forêt et Trame écologique ». Séquence pédagogique ENSP Versailles $3^{\mathrm{e}}$ année (octobre 2015, Alpes Mancelles, département de la Sarthe).

\subsection{Paysage dessiné vs paysage numérisé}

La démarche d'observation empirique et inductive encore bien présente dans les écoles de projet explique l'usage fréquent de représentations graphiques traditionnelles : croquis dessinés à la main, dessin paysager, bloc-diagramme, maquette grand format en deux ou trois dimensions, etc. La connaissance scientifique rationaliste est passée au filtre de l'utilité et de l'intérêt : des réticences sont ainsi formulées sur des techniques numériques réduisant le paysage à la tyrannie de l'image.

Pourtant, des gestionnaires électroniques de données permettent aujourd'hui de disposer de véritables systèmes d'informations géographiques (SIG), utilisables à volonté par la collecte et le traitement systématique des données géonumérisées (Brossard et Wieber, 2008).

Des logiciels comme QGIS ou ArcView facilitent le stockage de l'information et offrent la possibilité de naviguer dans d'importantes collections d'images ; ils permettent de structurer l'information de manière très complète et $\mathrm{d}$ 'accéder aux documents par requêtes très sophistiquées (Ormaux, 2005). De plus, il est possible par extraction de sélectionner des données, d'opérer des traitements statistiques, et de réaliser des mesures sur des objets spatiaux préalablement identifiés par l'ordinateur. En écologie, il est ainsi possible d'estimer précisément l'emprise spatiale d'un corridor, mesurer leur longueur, repérer des connexions, calculer des surfaces, ou encore déterminer au moyen d'indices l'hétérogénéité ou la fragmentation du paysage (Figure 4). Les coupes, transects ainsi que toutes les vues en perspective se prêtent à des traitements numériques afin de rendre compréhensible par tous des structures paysagères. Des modèles numériques donnent la possibilité de faire des simulations et de jouer sur des variables discriminantes afin de faire évoluer ces structures. 
Cette écologie du paysage invente ainsi une nouvelle organisation des systèmes écologiques et mobilise des savoirs techniques au service de la démarche du projet de paysage. L'outil se révèle efficace pour analyser des mosaïques paysagères complexes, mais son approche éloignée du sensible fait débat et soulève quelques questionnements.

En effet, si la carte fabriquée sur ordinateur autorise d'innombrables possibilités de traitement de l'information géographique, elle bouleverse aussi les expériences du visible et les mécanismes par lesquels se construit une image de la réalité. Une image produite sur écran ne retranscrit pas le paysage tel qu'il a été vu et dessiné sur le terrain. L’image modélisée est pour beaucoup de concepteurs une image aseptisée, une construction mathématique qui fabrique un paysage à distance, par le biais d'un truchement : un modèle abstrait donnant en même temps l'illusion du projet, puisqu'il produit des formes à partir du croisement d'un ensemble de données qui obéit à des normes et dont surtout le sens échappe aux élèves.
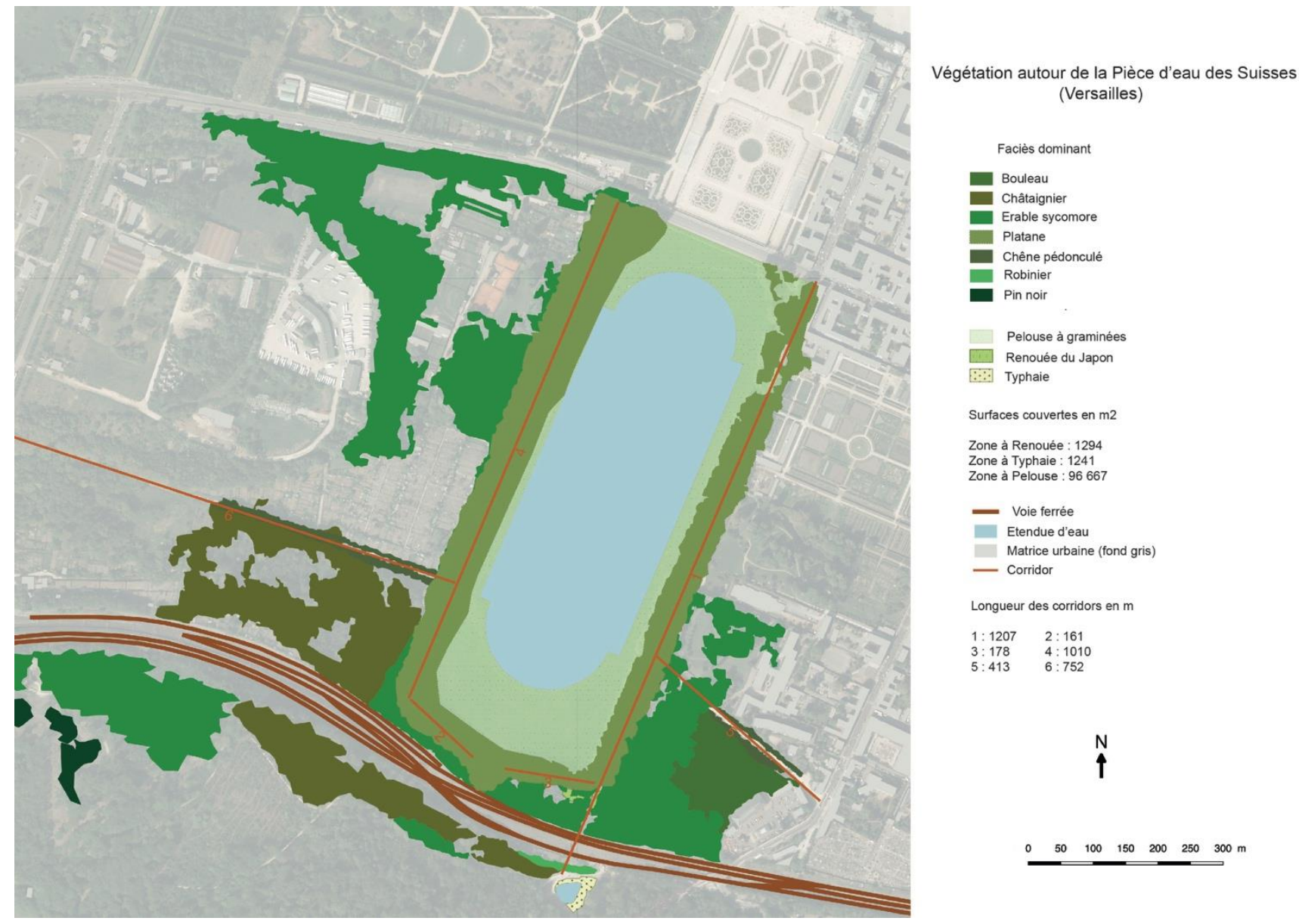

Figure 4. Paysages et informations géonumériques

Carte réalisée par le logiciel QGIS à partir des relevés de terrain et de l'utilisation des bases de données Corine Land Cover, BD TOPO, BD ORTHO

Aussi, le paysagiste qui prend plaisir à capter la réalité mouvante d'un paysage et à croquer sur le vif l'expérience de la matière ne s'y retrouve pas. L'emploi d'un SIG peut brider le concepteur dans ses choix graphiques toujours très personnels. Par ailleurs, la technologie numérique retranscrit difficilement la composante idéelle du paysage qui renvoie à la subjectivité. C'est pourtant cette composante qui fait sens pour le concepteur car elle est l'expression d'un ressenti et permet d'accorder des valeurs aux paysages, c'est-à-dire des préférences qu'une personne peut avoir pour une vue, une ambiance ou un agencement de formes particulières. Pour faire face à ces critiques, il importe aux enseignants de bien préciser les limites de ces outils mais également la finalité du 
travail demandé. Pour y parvenir, il est alors nécessaire de requestionner les modes de production des données et de réfléchir à des mises en situation permettant de renouveler l'approche paysagère.

\section{Ecologie et nouvelle pensée paysagère}

\subsection{Réinventer des modes de production et de représentation paysagère}

Inventer de nouveaux modes de production et de représentation des paysages pose la question des méthodes d'apprentissage pour pouvoir y parvenir. Quels apprentissages développés dans une école de projet ? Et quels sens donner aux représentations dans une démarche de projet ?

La démarche inductive très pratiquée dans les écoles de projet conduit à une approche essentiellement descriptive liée à l'observation du site. Les documents consultés ou produits sur un carnet de note partent du réel en faisant croire qu'il dit d'emblée des choses, qu'il est signifiant en lui-même, alors qu'ils sont en fait déjà la marque d'une construction de la pensée liée à l'analyse ou à l'interprétation que l'on s'est faite à partir de la lecture du site. En écologie, l'induction peut conduire l'étudiant à une exploration minutieuse du site : les plantes sont identifiées, les principales structures paysagères sont dessinées, les ressources locales faisant « matières à projet » sont recensées. L'approche permet de saisir en situation réelle l'existant et de diagnostiquer des potentialités susceptibles de s'exprimer dans le cadre d'un futur projet. Si cette approche est séduisante, c'est qu'elle laisse s'exprimer aussi l'imaginaire en hybridant des approches naturalistes et sensibles, invitant à lire dans les structures paysagères des changements. Ceux-ci résultent de la combinaison de facteurs (biophysiques, sociaux) mais surtout de la capacité qu'ont les hommes à considérer un espace dans une dimension poétique, sensible et créatrice, donnant corps au paysage et au projet. Cette façon de faire n'est pas celle des écologues qui ont tendance à privilégier une approche déductive et matérialiste par laquelle le paysage correspond à la résultante de la dynamique du milieu et de la société qui s'y est établie (Burel et Baudry, 1999, Clergeau, 2007). Dès lors, la lecture d'un espace est bien plus portée sur le cadre biophysique (topographie, géomorphologie, pédologie, végétation) permettant d'aborder l'analyse des formes du paysage et des différentes utilisations du sol. Lorsque cette lecture est historique, elle permet d'évaluer les changements (analyse diachronique) et amorce aussi une réflexion sur l'évolution de la fonctionnalité des milieux et les raisons qui la sous-tendent. L'approche déductive s'accompagne généralement d'une hypothèse qui en fait une méthode scientifique afin d'en déduire des conséquences observables futures, mais également passées, permettant d'en déterminer la validité. Confrontée au terrain de l'expérience, la déduction peut éclairer certains faits de nature que l'on aura du mal à expliquer : la morphologie complexe d'un relief, le dessin mouvementé d'un cours d'eau, la stratégie adaptative et évolutive d'une plante, la dynamique d'une communauté végétale ou animale.

L'un des enjeux de la formation consiste donc à montrer aux étudiants que la démarche inductive ne s'arrête pas à la description et à l'observation mais qu'elle mène aussi à une abstraction, qui soustend la mise en place ou le réinvestissement d'un vocabulaire spécifique et de notions indispensables à l'analyse et à l'explication des phénomènes et des mécanismes qui sont en jeu dans l'organisation spatiale.

Dans la pratique, il est tout à fait possible de combiner induction et déduction car les deux démarches ouvrent des voies de raisonnements complémentaires et sont adaptées à la construction d'une pensée complexe amenant l'apprenant à alterner entre le local et le global, le réel (in situ) et l'abstraction, l'expérience et le modèle de référence. Le projet doit pouvoir articuler ces échelles et ces façons de produire des connaissances qui devront être discutées en groupe et confrontées à la 
nécessité de leur efficacité opérationnelle. La démarche itérative communément adoptée dans les écoles de paysage facilite cet apprentissage d'autant plus que le paysage autorise " l'indiscipline » ou facilite les passerelles pour communiquer entre les disciplines.

Renouveler l'approche paysagère n'est donc possible que dans le cadre de séances collectives basées sur l'interaction entre les apprenants avec des temps de travail où l'on s'interroge sur le sens des connaissances produites accompagnant les représentations.

\subsection{Renouveler l'approche paysagère}

Le projet de paysage ne devient véritablement opérationnel que s'il s'inscrit dans une dynamique d'échange des savoirs entre les différents participants du projet. Les cartes, en tant que medium, peuvent très bien être conçues de façon collective afin qu'elles soient porteuses d'un projet partagé. Dans le cadre d'ateliers participatifs, il est ainsi possible d'inviter des acteurs locaux à réagir à des cartes et bases de données SIG fabriquées par les scientifiques car ces cartes « expertes » sont porteuses d'informations qu'ils vont apprendre à décrypter; inversement, on peut leur demander de réaliser à leur tour des représentations cartographiques susceptibles de révéler des enjeux environnementaux ou paysagers qui peuvent être très différents selon les acteurs concernés. Les paysagistes pourront se nourrir ainsi de ces différentes informations pour construire et définir la pertinence de leur projet.

Parallèlement, puisque le projet se construit par un va-et-vient permanent où se discute et se partage l'information, il paraît logique d'accorder autant de place aux représentations lues qu'aux représentations que l'on réalise. Les acquisitions liées aux lectures se font parallèlement à l'apprentissage des réalisations ; cette mise en situation oblige les apprenants à les questionner tout au long du processus de projet en justifiant leurs choix. Cette approche favorise la construction progressive du langage graphique, son appropriation, mais aussi son articulation avec d'autres langages propres à la culture des paysagistes.

Inversement, lorsque des ateliers participatifs sont organisés, il est tout à fait possible de reconstruire ce qui est une expérience personnelle en objet de réflexion et d'élaborer des moments où l'on s'interroge sur le sens des représentations. Un document réalisé par un intervenant peut être déconstruit et recontextualisé afin de comprendre le cheminement de la pensée de son auteur et son intention.

Enfin, appliquée à l'écologie, le renouvellement de l'approche paysagère amène à refonder une relation avec la nature en la considérant comme un tout composée d'êtres vivants humains et non humains. L'injonction permanente à évaluer les fonctions et les services qu'elle rend est révélatrice d'une culture managériale et d'une volonté récurrente d'apporter une ligne de conduite aux espaces de nature avec des usages connectés à des réglementations mais souvent déconnectés des pratiques qui naissent à la livraison du projet puis évoluent au quotidien avec les usagers. Qu'en est-il de ces pratiques et doit-on associer les usagers au suivi écologique des sites ? Les enjeux écologiques définis au moment des commandes et qui ont orientés les projets sont-ils les mêmes au bout de 5,10 ou 20 ans ? Sont-ils compris et acceptés par les usagers? Si la science écologique doit aider à mieux comprendre les évolutions d'un site et donc les dynamiques du vivant, elle se doit aussi de rester au contact des mutations territoriales et d'être au plus proche du citoyen. Cette science facilitatrice pourra ainsi rapprocher - par la culture de dialogue - les acteurs afin de construire des problématiques émergentes et les projets de demain.

L'écologie attachée à des concepts, à des modèles, et à une forme d'ingénierie environnementale se doit donc d'évoluer vers un nouvel objet scientifique en se jouant des frontières et en capitalisant les fruits de pratiques transgressives redéfinissant le paysage lui-même et les représentations des 
acteurs du territoire, telles que les révèlent « à chaud » les rencontres organisées directement sur le terrain ou dans un contexte plus formalisé d'ateliers.

\section{Bibliographie}

Arnould P., «Un jardin dans la ville - Quelle biodiversité urbaine pour demain ? », Territoire en mouvement [En ligne], $\mathrm{n}^{\circ} 12,2012$, URL : http://tem.revues.org/1436

Arnould P., Au plaisir des forêts, Paris, Editions Fayard, 2014.

Barrès P., « Pratiques du site, écologie du lieu », Marges [En ligne], $\mathrm{n}^{\circ}$ 14, 2012, URL : http://journals.openedition.org/marges/298

Beroutchachvili N., Rougerie G., Géosystèmes et paysages, Bilan et méthodes, Paris, Editions A. Colin, 1991.

Brossard T., Wieber J.-C. (dir.), Paysage et information géographique, Paris, Editions Lavoisier, hermes-sciences publications, 2008.

Brunet R., Ferras R., Théry H., Les mots de la géographie, Paris, Editions Reclus-La Documentation Française, 1992.

Burel F., Baudry J., Ecologie du paysage. Concepts, méthodes et applications, Paris, Editions Tec et Doc, 1999.

Chauvel G., Rumelhart M., «Phytosociologie et jardin : vers une éthologie végétale ? Pour l'intégration, dans les relevés phytosociologiques, des tactiques d'occupation et de conquête de l'espace par les végétaux (cas des phytocénoses horticoles) », Actes du XVII ${ }^{\mathrm{e}}$ colloque phytosociologique : Phytosociologie et paysage, 1988, Berlin, Editions Cramer, p. 47-92.

Clément G., Lonsdale M., Pelt J.-M., Scheyder P., Des jardins et des hommes, Montrouge, Editions Bayard, 2016.

Clergeau P., Une écologie du paysage urbain, Paris, Editions Apogée, 2007.

Corajoud M., Le paysage c'est l'endroit où le ciel et la terre se touchent, Editions Actes Sud, 2010.

Donadieu P., Sciences du paysage, Entre théories et pratiques, Paris, Editions Lavoisier, 2012.

Hobbs R.-J., Higgs E., Harris J.-A., « Novel ecosystems: implications for conservation and restoration », Trend in ecology an evolution, vol. 24, $\mathrm{n}^{\circ} 11,2009$, URL : http://www.ces.fau.edu/climate_change/evergladesrecommendations-2014/pdfs/session-a-resource-2.pdf

Kalaora B., « Naissance et développement d'un loisir urbain : la forêt de Fontainebleau », Etudes rurales, 1981, vol. $83, \mathrm{n}^{\circ} 1, \mathrm{p} .97-115$.

Lévêque C., L'écologie est-elle encore scientifique?, Paris, Editions Quæ, 2013.

Lévêque C., La biodiversité, avec ou sans l'homme ? Versailles, Editions Quæ, 2017.

Luginbühl Y., La mise en scène du monde. Construction du paysage européen, Paris, Editions CNRS, 2012.

Michelin Y., «Le bloc-diagramme : une clé de compréhension des représentations du paysage chez les agriculteurs ? Mise au point d'une méthode d'enquête préalable à une gestion concertée du paysage en Artense (Massif central français) », Cybergeo : European Journal of Geography, n 118, 2000, URL : http://cybergeo.revues.org/1992

Mollie C., Des arbres dans la ville : l'urbanisme végétal, Paris, Editions Actes Sud, 2009.

Mottet J. (dir.), La forêt sonore. De l'esthétique à l'écologie, Paris, Editions Champ-Vallon, 2017.

Ormaux S., 2005, « Le paysage entre l'idéel et le matériel », in Droz Y., Miéville-Ott V. (dir.), La polyphonie du paysage, Lausanne, Editions Presses polytechniques et universitaires romandes, p. 71-99.

Petit-Berghem Y., Écologie et paysage. Réinterroger le vivant. Toulouse, UPPR Editions, ISBN : 978-2-37168-064-7, https://www.uppreditions.fr/livre/978-2-37168-064-7 ecologie-et-paysage-reinterroger-le-vivant/, 2016.

Roger A., Court traité du paysage, Paris, Editions Gallimard, 1997, 216 p. 